\title{
ANALYSIS OF THE LONGITUDINAL LOW-ORDER MODE BEAM DYNAMICS IN PEP-II RINGS AT HIGH CURRENT BEAMS *
}

\author{
T. Mastorides ${ }^{\dagger}$, C. Rivetta ${ }^{\ddagger}$, J.D. Fox, D. Teytelman, D. Van Winkle \\ Stanford Linear Accelerator Center \\ Stanford, CA 94309, USA
}

\begin{abstract}
PEP-II plans to achieve the final goal in luminosity will require an increase of the beam currents to $4 \mathrm{~A}$ for LER and 2.2A for HER. These magnitudes are challenging in part because they will push the longitudinal low-order mode (LOM) beam stability and the station stability to the limit. To analyze the behavior of both rings at high currents and to understand the limits in the longitudinal feedback systems, a simulation tool has been developed at SLAC. This tool is based on a reduced model of the longitudinal LOM dynamics of the beam interacting with the effective impedance presented by the RF stations. Simulations and measurements of the longitudinal beam behavior in both rings have been performed to understand the ultimate limits of the systems. These studies have defined the impact of control loop parameters in the longitudinal beam dynamics, identified the limiting behavior of RF devices affecting the optimal performance of the RF stations and quantified the behavior of the longitudinal LOM beam dynamics. Results of sensitivity to parameter variations in the beam dynamics and limits in the maximum current that LER/HER can achieve based on the longitudinal beam stability are reported in this paper.
\end{abstract}

\section{SIMULATION}

The simulation uses a reduced model of the PEP-II RF system [1] to capture the low order mode dynamics through the beam interaction with the cavities and the fast LLRF feedback loops. The simulation has been validated with the real system using the same tools to extract the growth/damping rates of the low order modes from both systems [2]. The simulation has been used to study parameter sensitivity and to predict ultimate limits. It has also been fundamental in understanding limitations of the technology, implementation details/imperfections and their effect on beam stability. Earlier studies, results and conclusions are available in [3].

\section{DEFINITION OF LIMITS}

In this paper, we study three kinds of limitations on operating conditions: the RF station stability limit, the klystron static power requirement, and the dynamic stability limit through the longitudinal growth rates of the particle beam.

\footnotetext{
* Work supported by the U.S. Department of Energy under contract \# DE-AC02-76SF00515

$\dagger$ themis@ slac.stanford.edu

‡ rivetta@slac.stanford.edu
}

The RF system can be considered as a multiple input, multiple output system. Due to the klystron saturation, the gain for the two directions (phase and amplitude modulation) changes differently with operating point. Therefore, to satisfy the same gain and phase margins as the operating point moves into saturation, the performance is reduced. The RF station stability is related with the magnitude of the klystron saturation and depends on the operating point defined by the RF station parameters.

The klystron static power limit is set lower than the maximum specified power to accommodate for the high voltage power supply ripple, the collector dissipated power and the line perturbations. Their collective margin amounts to a $15-20 \%$ reduction of the maximum available klystron power. Individual klystron data and non-linearities for each station and klystron type (Phillips, Marconi and SLAC) are included in the simulation to accurately predict the klystron power limits.

The beam stability depends on the operation point. At high currents and with the minimum impedance achievable by the impedance control tools, the beam is naturally unstable without the damping feedback loop. The comparison of the beam's instability growth rates with the available damping determine the stability of the system.

Successful operation of the machine requires all three conditions to be met concurrently. Trade-offs among them exist and may be necessary at higher currents as will be shown.

The predictions presented in this paper are not hard limits; operating points past which it will be impossible to run the machine. Rather, they are operating points where one or more of the design margins are lost, leading to reduced performance and unsustainable configurations. Since this happens for a relatively ideal system (the simulation), it will be worse for the real machine causing an increased probability of aborts. Such a condition was reached at the end of Run 5, when a small increase in current in the LER, led to a substantial increase in the rate of aborts.

\section{HER LIMITATIONS}

\section{RF Station Stability}

Fig. 1 shows the power curves of a 1.2 MW Marconi klystron as used in the LER. To run consistently in a relatively linear region, the input power is kept close to $15 \mathrm{~W}$. An increase of output power above $800 \mathrm{~kW}$ via increases in input power, leads into saturation and ultimately reduces RF station stability as explained above. Therefore, 


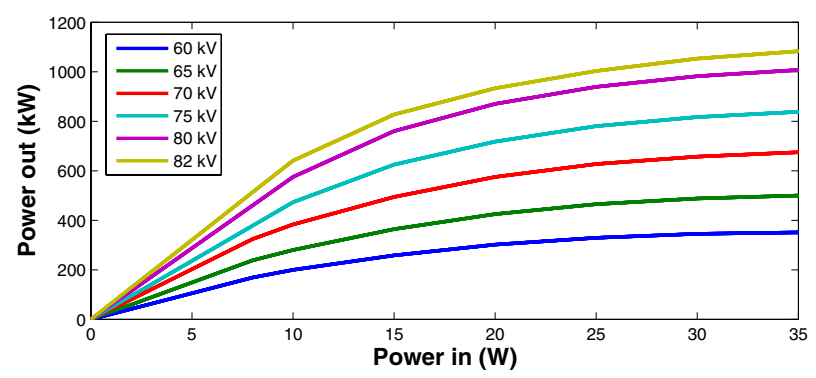

Figure 1: Power Curves for Marconi \#4 klystron.

the maximum klystron forward power is operationally unsustainable since the gain goes to zero. The effect of the saturation can also be seen from the distortion in the closed loop frequency response of the system - indicating the reduced margins of the feedback loops - as shown in Fig. 2. Considerations of loop and station stability - our

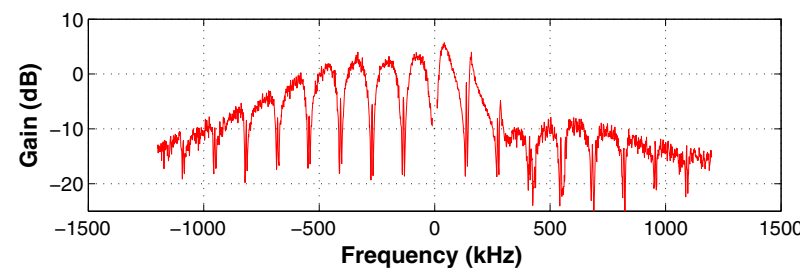

Figure 2: Frequency response distortion at high current.

ability to optimally tune the stations - set the power limits to $\approx 1030 \mathrm{~kW}$ for SLAC klystrons and $\approx 930 \mathrm{~kW}$ for Phillips/Marconi klystrons.

\section{Klystron Power Limit}

The dominant limitation for the HER is the available forward klystron power. A careful balance of power between the 2 and 4 cavity stations is necessary to maximize the power margin for both. From these considerations the power limit is set to $\approx 1120 \mathrm{~kW}$ for SLAC klystrons and $\approx 960 \mathrm{~kW}$ for Phillips/Marconi klystrons. Since the station stability limit is lower, it will be used for our analysis. Fig. 3 shows the distribution of forward power in the 2 and 4 cavity stations for a range of gap voltages (for constant voltage per cavity), as well as our limit of $1030 \mathrm{~kW}$. From this graph, we can see that the optimal gap voltage

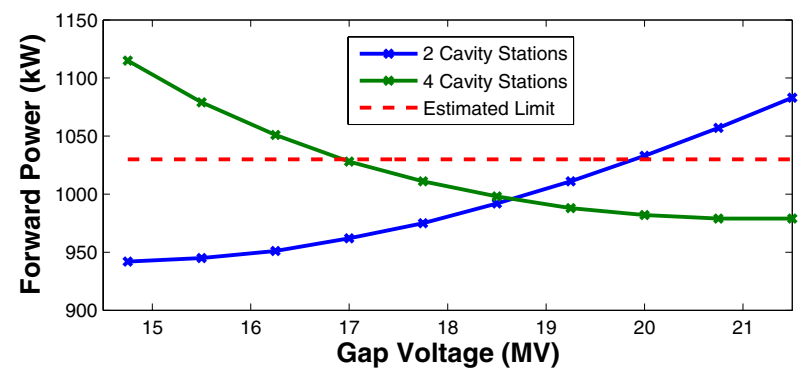

Figure 3: HER Power distribution versus Gap Voltage for $2.2 \mathrm{~A}$.

for $2.2 \mathrm{~A}$ is $18.65 \mathrm{MV}$, with $995 \mathrm{~kW}$ of forward power required from all stations. The optimal gap voltage (green) and the required klystron power (blue) are plotted versus current in Fig. 4. From this analysis, we see that our power limit is crossed at about $2.25 \mathrm{~A}$.

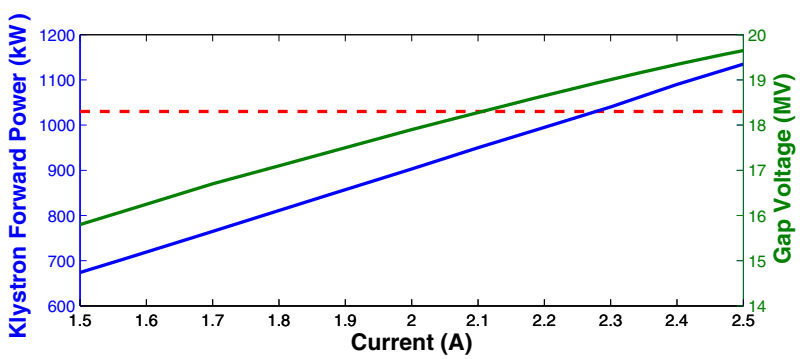

Figure 4: Optimal HER Gap Voltage and minimum necessary Klystron Forward Power versus Current.

\section{Growth Rates}

The growth rates of the low order modes $\sigma_{l}$ are compared with the damping rates $d_{l}$ to compute the effective damping rate $\Delta_{l}=d_{l}-\sigma_{l}$. From our experience with the practical and operational limits of control of the beam instability feedback and to allow for fluctuations of the growth rates due to drifts of parameters, the limit is set to $\Delta_{l} \mid=2 * \sigma_{l}$. The maximum available effective damping rate for the HER is $-3 \mathrm{~ms}^{-1}$ which sets the maximum operational growth rate to $1.5 \mathrm{~ms}^{-1}[4]$. With the same analysis, the damping rates for the LER are $-6 \mathrm{~ms}^{-1}$ and the maximum operational growth rate is $3 \mathrm{~ms}^{-1}$.

Fig. 5 shows the comparison of the growth rates extracted from the simulation data in blue, with the average of the experimentally measured growth rates in the physical machine in magenta (for a gap voltage of $16 \mathrm{MV}$ ). One can also see the big variance of the measured growth rates which necessitate the margins defined above. The optimal configuration curve is at the planned 18.5 $\mathrm{MV}$ and includes the improved performance of the more linear driver amplifiers ${ }^{1}$. From this curve, it is determined that the HER LOM growth rates do not exceed our estimated limit, even for the highest planned current of $2.2 \mathrm{~A}$.

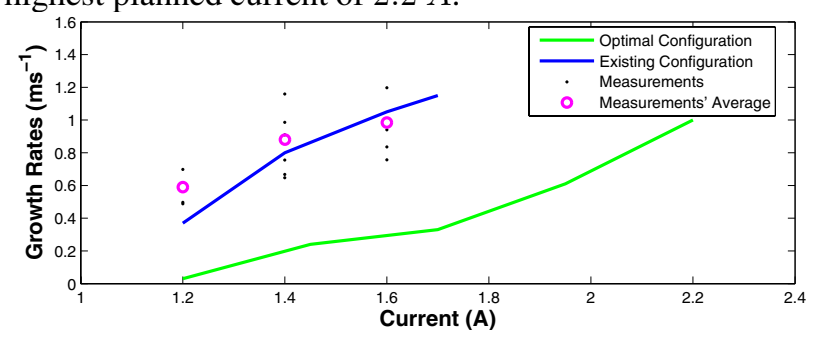

Figure 5: HER Growth Rates.

\section{LER}

\section{$R F$ Station Stability}

The analysis for the RF station stability is the same as for the HER, leading to a power limit of $\approx 1030 \mathrm{~kW}$ for SLAC klystrons and $\approx 930 \mathrm{~kW}$ for Phillips/Marconi klystrons.

\section{Klystron Power Limit}

The LER situation is more difficult because all three limitations are reached. Fig. 6 shows the required

\footnotetext{
${ }^{1}$ Amplifiers with flatter frequency response are installed following simulation studies of the effect of the existing amplifiers' non-idealities on machine performance.
} 
klystron forward power versus current. With the existing Phillips/Marconi klystrons the limit is crossed at $3500 \mathrm{~mA}$ with $4.05 \mathrm{MV}$ gap voltage and at $3650 \mathrm{~mA}$ with $4.5 \mathrm{MV}$.

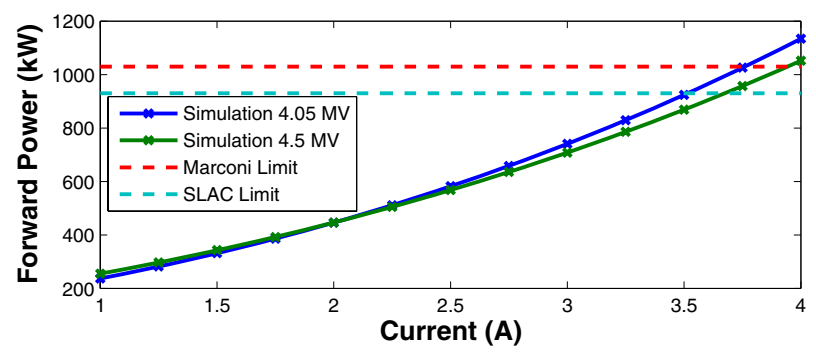

Figure 6: LER Klystron Forward Power versus current.

If all klystrons in the LER were to be SLAC klystrons, the limits from power considerations become $3750 \mathrm{~mA}$ and $3900 m A$ respectively.

To examine the feasibility of the $4 A$ case, the forward power is plotted versus gap voltage in Fig. 7. For 3.6 A, at least $4.4 \mathrm{MV}$ are needed. $4 \mathrm{~A}$ cannot be achieved with the

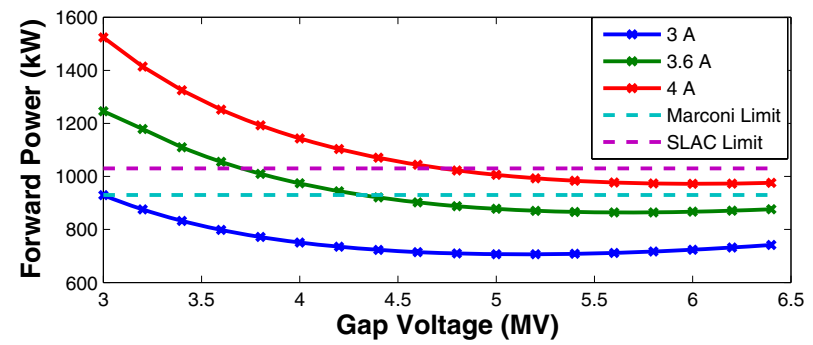

Figure 7: LER Klystron Forward Power versus Gap Voltage.

existing klystrons. SLAC klystrons will be required in all the LER stations, together with an increase of gap voltage to at least $5 \mathrm{MV}$ for sufficient margin. At this increased gap voltage, problems with vacuum chamber heating and high order mode issues related to the shorter bunch length may arise.

\section{Growth Rates}

The LER growth rates pose a significant limitation to the maximum attainable current. Fortunately, there are certain upgrades and new algorithms that can significantly improve the limit. These include SLAC klystrons, the comb phase rotation (a partially implemented trade off of RF station stability to growth rate improvement)[3] and the use of more linear LLRF drive amplifiers[5]. From Fig. 8 and Fig. 9

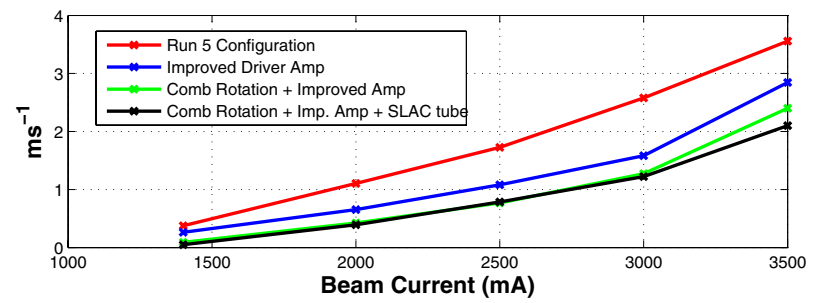

Figure 8: Estimated Growth Rates at 4.05 MV.

we can predict that with the existing RF station implementation, the limit due to growth rates is at about $3100 \mathrm{~mA}$.

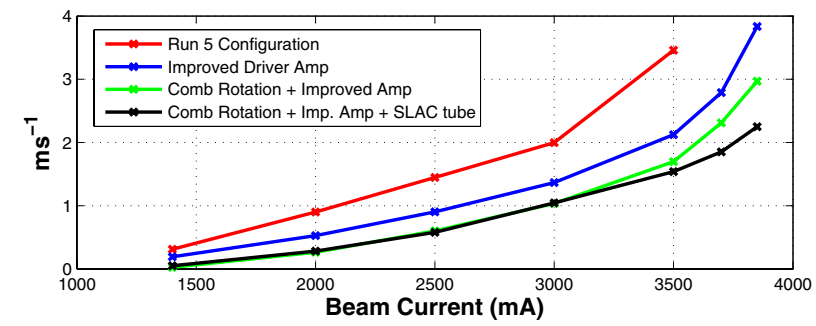

Figure 9: Estimated Growth Rates at 4.50 MV.

The power limit of $3500 \mathrm{~mA}$ can be reached with a combination of the upgrades. An increase of gap voltage to $4.5 \mathrm{MV}$ raises the limit of the existing system to $3250 \mathrm{~mA}$, with a combination of the improvements to $3600 \mathrm{~mA}$ and with all the improvements and the SLAC klystrons to the power limit of $3900 \mathrm{~mA}$.

\section{CONCLUSIONS}

This simulation of the PEP-II rings is a close representation of the actual system. It has been used to study the existing system and planned upgrades. It can also be used to study other suggested configurations.

These studies suggest that the HER could achieve $2.2 \mathrm{~A}$, but higher currents will be very difficult to reach due to power limitations. For the LER with the existing LLRF station implementation and an increased gap voltage of $4.5 \mathrm{MV}$ we expect a $3250 \mathrm{~mA}$ limit. The improved amplifiers and the comb rotation raise this limit to $3650 \mathrm{~mA}$. Finally, SLAC klystrons for all LER stations and a gap voltage higher than $5 M V$ are needed for $4 \mathrm{~A}$, with possible problems with vacuum chamber heating and issues related to higher order modes.

\section{ACKNOWLEDGEMENTS}

The authors would like to thank the klystron department for their help with klystron tests and the PEP-II operations for providing us with machine time for measurements. We would also like to thank Heinz Schwarz for the helpful discussions of static power limits.

\section{REFERENCES}

[1] P. Corredoura. "Architecture and Performance of the PEP-II Low-Level RF System”, SLAC-PUB-8124, March 1999.

[2] J. Fox et. al., "Multi-Bunch Instability Diagnostics Via Digital Feedback Systems at PEP-II, DA $\Phi$ NE, ALS and SPEAR", SLAC-PUB-8128, June 1998.

[3] C. Rivetta et. al., "Modeling and Simulation of Longitudinal Dynamics for Low Energy Ring-High Energy Ring at the Positron-Electron Project", Phys. Rev. ST-AB, 10, 022801 (2007).

[4] D. Teytelman et. al., "Operating Performance of the Low Group Delay Woofer Channel in PEP-II", SLAC-PUB11253, May 2005.

[5] J. Fox et.al, "Selecting RF Amplifiers for Impedance Controlled LLRF Systems - Nonlinear Effects and System Implications", PAC 2007. 